\title{
Dietary vitamin E and physical exercise: I. Altered endurance capacity and plasma lipid profile in ageing rats
}

\author{
S. Asha Devi ${ }^{\mathrm{a}, *}$, S. Prathima ${ }^{\mathrm{a}}$, M.V.V. Subramanyam ${ }^{\mathrm{b}}$ \\ ${ }^{a}$ Laboratory of Gerontology, Department of Zoology, Bangalore University, Bangalore 560056, India \\ ${ }^{\mathrm{b}}$ Department of Sericulture, Bangalore University, Bangalore 560 056, India
}

Received 22 July 2002; received in revised form 16 October 2002; accepted 18 October 2002

\begin{abstract}
The effect of vitamin $\mathrm{E}$ on the exercise performance and plasma lipid profile was studied in male Wistar rats of 4-(young adults), 8-(old adults), 12-(middle-age) and 22-months (old) of age. Animals were orally supplemented with vitamin E and allowed to swim for 30 min/day, 5 days/week and for a total period of 60 days. Swim velocity $\left(S_{\mathrm{v}}\right)$, external work done $\left(W_{\text {ext }}\right)$ and endurance $(E)$ capacity were the parameters that were used to assess the exercise performance of the trained rats that were either supplemented or non-supplemented with the dietary antioxidant. Plasma lipid profile analyses were in terms of low-density lipoprotein (LDL-C), high-density lipoprotein, (HDL-C) cholesterol and total cholesterol (C). Age-related decline in $S_{\mathrm{v}}$ was noticeable in the 22-months old rats. However, the effect of vitamin E on the $S_{\mathrm{v}}$ between the trained groups was not evident in any of the age groups. $W_{\text {ext }}$ increased linearly with age with no significant variations between the trainees. Trainee rats, when allowed to swim to exhaustion, showed a higher endurance capacity when supplemented with vitamin E. However, this capacity declined with age. There was a significant age-associated elevation in plasma $\mathrm{C}$ with corresponding increase in LDLC. Exercise training in conjunction with vitamin E supplementation was most effective in elevating HDL-C levels in all age groups. These changes were accompanied by significant reductions in cholesterol/HDL-C ratios in animals receiving vitamin E, sedentary or otherwise. Our data suggests that it may be important to consider vitamin $\mathrm{E}$ while attempting to derive the benefits of swim training, both in terms of favorably altering the plasma lipid profile as well as enhancing the endurance capacity of exercise trainees. Dietary supplementation by vitamin E could attenuate the early onset of fatigue in the old.
\end{abstract}

(C) 2002 Elsevier Science Inc. All rights reserved.

Keywords: Aging; Cholesterol; Endurance; Exercise; High-density lipoprotein; Low-density lipoprotein; Vitamin E

\section{Introduction}

Physical training allows an animal to successfully respond to exercise of varied intensities. Also trained animals can sustain intense activity for a longer period before fatigue sets in as compared to untrained animals (Venditti and Meo, 1996). Vitamin E has been examined as an ergogenic aid in studies that are performed at an altitude of $6000 \mathrm{ft}$ (Van Der Beek, 1991) and also for its role in protection against development of age-related diseases such as atherosclerosis (Chan, 1998). While exercise is known to alter lipid profile favorably, its effect when combined with an antioxidant such as vitamin E, needs to be examined. $\alpha$-tocopherol $(\alpha-\mathrm{TocH})$ is biologically and chemically the most potent form of vitamin $\mathrm{E}$ and is a major

\footnotetext{
* Corresponding author. Tel.: +91-80-3214011x214.

E-mail address: asuba@blr.vsnl.net.in (S. Asha Devi).
}

free-radical-trapping antioxidant in plasma low density lipoprotein (LDL) and is therefore the most sought after lipid soluble antioxidant compared to $\gamma$-TocH, retinal and carotenoids (Bowry et al., 1992). Exercise, however, when performed strenuously, is associated with increased production of reactive oxygen species (ROS) which eventually damage biological molecules and key cellular components (Polidori et al., 2000). Therefore, a balance between the beneficial and harmful effects of exercise, may be of importance, particularly in the elderly.

The present study, while addressing the ergogenic benefits of vitamin $\mathrm{E}$ in trainee animals has also attempted to evaluate pertinent age-related responses in terms of (i) extent of trainability in the young vs. old rats supplemented with vitamin $\mathrm{E}$ or otherwise (ii) impact of training in enhancing the swim performance through the endurance capacity of the trained animals, adjunctively supplemented 
with the antioxidant. These results are likely to validate our hypotheses that (i) swim endurance can be prolonged with the administration of vitamin E. (ii) the possibility of a shift of the plasma lipid profile favorably towards high density lipoprotein (HDL) may serve as a good tool to evaluate exercise effectiveness in the supplemented trainees.

\section{Materials and methods}

The present study was approved by the Institutional Animal Ethics Committee (IAEC), Bangalore University, India.

\subsection{Animal care and training protocol}

Male pathogen-free albino rats of Wistar strain of 4 months (months) were obtained from the Central Animal Facility, IISc, Bangalore, and were maintained until they were of 8,12 and 22 months in a clean rodent room without the SPF status being monitored. However, animals from outside were not allowed and the room was exclusively meant for maintenance of aging rats. The maximum length of life of male Wistar rats in our animal colonies has been 30 months with the median length of 15.5 months. The experiments were completed taking into consideration the whole age-scale encompassing the young adult, old adult, middle-aged and the old. They were housed 2-3 per cage in cages that were fitted with stainless-steel wire-mesh bottoms, maintained at the temperature of $28 \pm 1{ }^{\circ} \mathrm{C}$, relative humidity of $77.5 \pm 1 \%$, and under daily photoperiods of $12 \mathrm{~h}$-light and $12 \mathrm{~h}$-dark cycle. The animals were fed with laboratory chow (Lipton India Ltd, Bangalore) and tap water ad libitum. Rats were assigned to (A) two sedentary groupssedentary controls on a normal diet (SE-C, N) and sedentary controls on vitamin $\mathrm{E}$ supplementation (SE-C, $+\mathrm{E}$ ), and, (B) two exercise groups-swim trainees on a normal diet (SW-T, N) and swim trainees on vitamin $\mathrm{E}$ supplementation (SW-T, +E). Exercise training was similar to the protocol described by Prathima and Asha, (1999) with minor modifications. Briefly, the animals were exercised in a glass tank $(77 \times 38 \times 39 \mathrm{~cm})$ filled to a depth of $21 \mathrm{cms}$ for rats of 4 and 8 months, $25 \mathrm{cms}$ for those of 12 months and $31 \mathrm{cms}$ for those of 22 months. Rats were made to swim with a load of $3 \%$ of their body weight tied to their tails. Initially they were made to exercise for $5 \mathrm{~min} /$ day with a progressive increase to $30 \mathrm{~min} /$ day over a period of one week, and thereafter for $30 \mathrm{~min} /$ day, for a total training period of 12 weeks with five training days/week. Two groups of animals received a daily oral supplementation of $50 \mathrm{IU} / \mathrm{kg}$ of $\alpha$-tocopherol, throughout the training period. The group SE-C $(\mathrm{N})$ under (A) was restricted to only cage activity. There was no mortality in any of the groups in any age during the training and even after that.

\section{Swim parameters}

Swim velocity $\left(S_{\mathrm{v}}\right)$ : The distance traversed by the animals in unit time was measured and expressed in terms of $\mathrm{m} / \mathrm{min}$.

External work done $\left(W_{\text {ext }}\right)$ : The work done was calculated according to (Brooks and White, 1978) and expressed as $\mathrm{kg} \mathrm{m} / \mathrm{min}$.

Endurance test $(E)$ : Endurance capacities of swim trainees with or without vitamin $\mathrm{E}$ supplementation were assessed at the conclusion of training period by making them to swim to exhaustion. Exhaustion is defined according to the criterion of Dawson et al., (1968) as the point at which the rats remained below the water surface for $10 \mathrm{~s}$ and were recorded in minutes.

\section{Measurement of blood parameters}

Animals were anaesthetized (light) with ether and restrained in dorsal recumbency. The syringe needle was inserted just below the xyphoid cartilage and slightly to the left of midline. The needle was advanced at an angle of 10$20^{\circ}$ from the horizontal axis to the sternum for easy entry to the heart. Blood was carefully aspirated from the heart, mixed with $1 \%$ EDTA and centrifuged to separate the plasma.

Tissue sampling: All animals were sacrificed under light ether anesthesia. Preparation of left and right ventricles (LV and RV) was similar to our earlier protocol (Sharon and Asha Devi, 2001). Briefly, after withdrawal of the blood, the chest was opened with a midline incision and the heart was perfused with ice-cold Tyrode's buffer and rinsed several times with the same buffer. The organ was quickly weighed and the left and right ventricles were isolated. Regional weights were recorded after removing the interventricular septum.

High density lipoprotein-cholesterol (HDL-C): The HDL reagent provided in the kit (Rashmi Diagnostics, Bangalore, India) containing magnesium chloride was added to the plasma, mixed and left aside for $10 \mathrm{~min}$ at $25^{\circ} \mathrm{C}$ to allow the precipitation of chylomicrons, low density lipoproteins (LDL) and very low density lipoproteins (VLDL). Samples were centrifuged at $4000 \mathrm{rpm}$ for $1 \mathrm{~min}$. To the supernatant containing HDL-C, cholesterol reagent was added, mixed and incubated for $10 \mathrm{~min}$ at $37^{\circ} \mathrm{C}$. Absorbance was read at $505 \mathrm{~nm}$, and HDL-C was expressed as $\mathrm{mg} / \mathrm{dl}$.

LDL-C: LDL-C in the plasma was precipitated by addition of LDL reagent provided in the kit (Rashmi Diagnostics, Bangalore, India), vortex mixed and centrifuged at $4000 \mathrm{rpm}$ for $10 \mathrm{~min}$. Cholesterol in the supernatant was assayed by cholesterol reagent and the absorbance read at $505 \mathrm{~nm}$. Concentration of LDL-C was obtained from the difference between total cholesterol and cholesterol in the supernatant.

Total Cholesterol (C): Plasma was incubated with the cholesterol reagent (Rashmi Diagnostics, Bangalore, India) for $10 \mathrm{~min}$ at $37^{\circ} \mathrm{C}$ and absorbance measured at $505 \mathrm{~nm}$.

Statistical Analyses: All results are expressed as mean \pm SE Changes in body weights for the training 
Table 1

Summary of changes in body weights for the training period and dietary vitamin E supplementation in different age groups of rats

\begin{tabular}{|c|c|c|c|c|c|c|c|c|}
\hline \multirow[t]{2}{*}{ Age (months) } & \multirow[t]{2}{*}{ Groups } & \multicolumn{7}{|c|}{ Days of training } \\
\hline & & $\mathrm{PT}$ & 10 & 20 & 30 & 40 & 50 & 60 \\
\hline \multirow[t]{4}{*}{4} & SE-C(N) & $230 \pm 3.2$ & $242 \pm 3.6^{\prime \prime}$ & $250 \pm 3.1$ & $258 \pm 3.2$ & $266 \pm 2.6$ & $275 \pm 2.6$ & $286 \pm 3.4$ \\
\hline & SW-T(N) & $228 \pm 1.5$ & $232 \pm 5.8$ & $238 \pm 2.8$ & $243 \pm 6.1$ & $249 \pm 4.4$ & $255 \pm 3.2$ & $259 \pm 2.6$ \\
\hline & SE-C $(+E)$ & $230 \pm 3.6$ & $240 \pm 3.8^{\circ}$ & $252 \pm 1.1^{-}$ & $261 \pm 5.1$ & $269 \pm 5.0$ & $278 \pm 2.1$ & $284 \pm 2.0^{\prime \prime}$ \\
\hline & SW-T $(+E)$ & $228 \pm 6.8$ & $233 \pm 4.4$ & $239 \pm 3.6$ & $245 \pm 6.1$ & $250 \pm 3.1$ & $256 \pm 4.2$ & $261 \pm 3.6$ \\
\hline \multirow[t]{4}{*}{8} & $\mathrm{SE}-\mathrm{C}(\mathrm{N})$ & $316 \pm 3.0$ & $334 \pm 3.3^{-}$ & $343 \pm 3.1^{\circ}$ & $359 \pm 1.7^{-}$ & $370 \pm 4.1^{\prime \prime}$ & $381 \pm 4.6^{\circ}$ & $393 \pm 6.5$ \\
\hline & SW-T(N) & $317 \pm 3.0$ & $325 \pm 4.1$ & $329 \pm 3.3$ & $335 \pm 3.8$ & $348 \pm 4.5^{\prime \prime}$ & $358 \pm 3.3^{\circ}$ & $367 \pm 5.1$ \\
\hline & SE-C $(+E)$ & $317 \pm 4.3$ & $333 \pm 3.3^{-}$ & $343 \pm 3.8$ & $356 \pm 6.8^{\circ}$ & $366 \pm 4.2$ & $375 \pm 5.1$ & $390 \pm 2.6$ \\
\hline & SW-T $(+E)$ & $316 \pm 2.3$ & $322 \pm 2.1^{\circ}$ & $328 \pm 2.4^{\circ}$ & $334 \pm 3.5$ & $346 \pm 4.6^{\circ}$ & $355 \pm 4.1$ & $361 \pm 4.8$ \\
\hline \multirow[t]{4}{*}{12} & SE-C(N) & $364 \pm 4.6$ & $374 \pm 1.8^{\circ}$ & $386 \pm 1.8$ & $394 \pm 1.4^{-}$ & $400 \pm 2.1^{\prime \prime}$ & $410 \pm 1.1^{*}$ & $416 \pm 1.9^{\prime \prime}$ \\
\hline & SW-T(N) & $364 \pm 2.5$ & $366 \pm 2.0$ & $373 \pm 2.1^{\prime \prime}$ & $381 \pm 1.4^{-}$ & $386 \pm 1.4^{\prime \prime}$ & $394 \pm 1.2$ & $402 \pm 2.2$ \\
\hline & $\mathrm{SE}-\mathrm{C}(+\mathrm{E})$ & $362 \pm 2.4$ & $370 \pm 3.1^{\circ}$ & $382 \pm 2.9^{-}$ & $388 \pm 3.2$ & $393 \pm 3.2$ & $394 \pm 1.3$ & $409 \pm 1.6^{\circ}$ \\
\hline & $\mathrm{SW}-\mathrm{T}(+\mathrm{E})$ & $362 \pm 2.3$ & $368 \pm 2.3$ & $373 \pm 1.8$ & $380 \pm 1.1^{-}$ & $387 \pm 1.6^{-}$ & $394 \pm 1.3^{-}$ & $403 \pm 1.6^{-}$ \\
\hline \multirow[t]{4}{*}{22} & SE-C(N) & $503 \pm 1.5$ & $510 \pm 0.5^{-}$ & $521 \pm 1.5^{*}$ & $529 \pm 1.0$ & $537 \pm 2.5^{\prime \prime}$ & $544 \pm 2.2^{\circ}$ & $555 \pm 2.1^{-}$ \\
\hline & SW-T(N) & $507 \pm 1.5$ & $512 \pm 1.4$ & $517 \pm 1.1$ & $522 \pm 1.4^{\prime \prime}$ & $529 \pm 3.4^{\circ}$ & $535 \pm 2.0$ & $543 \pm 2.3^{\prime \prime}$ \\
\hline & SE-C $(+E)$ & $506 \pm 1.5$ & $512 \pm 1.1^{-}$ & $520 \pm 1.4$ & $528 \pm 1.4$ & $536 \pm 2.6^{-}$ & $543 \pm 2.2^{\prime \prime}$ & $551 \pm 2.3^{-}$ \\
\hline & $\mathrm{SW}-\mathrm{T}(+\mathrm{E})$ & $506 \pm 2.0$ & $510 \pm 1.3^{\circ}$ & $515 \pm 1.3$ & $521 \pm 1.0$ & $527 \pm 1.1$ & $532 \pm 4.0^{\prime \prime}$ & $539 \pm 2.4^{-}$ \\
\hline
\end{tabular}

Body weights are expressed as mean $\pm \mathrm{SE}$ of three animals/group. Significance of difference: ${ }^{-} P<0.0,{ }^{\circ} P<0.05,{ }^{\prime \prime} P<0.02, * P<0.001$ (student's $t$-test). Comparisons were made with respect to pre-training (PT) body weight. SE-C(N), sedentary control; SE-C(+E), sedentary control supplemented with vitamin E; SW-T(N), trained swimmers; SW-T(+E), trained swimmers supplemented with vitamin E; mon, months.

period and dietary supplementation of vitamin $\mathrm{E}$ was analyzed by Student's $t$-test. Heart weight (HW), body weight $(\mathrm{BW})$ and regional weights (LV and $\mathrm{RV}$ ) were analyzed by paired ' $t$ ' test. Other parameters were subjected to two-way ANOVA (groups $\times$ ages). Significant $F$ ratios between the group differences were further tested by Duncan's multiple range test (DMRT). Probability values of $P<0.05$ were considered significant (Snedecor and Cochran, 1994).

\section{Results}

Supplementation of vitamin E did not alter the food intake either in the sedentary animals or trainees. However, the trainees always exhibited higher intake of daily food by 3-4 g than their untrained counterparts that consumed 15$18 \mathrm{~g} /$ day. The average food consumption/day showed a decline with age in the 22 month-old animals, which was $10 \mathrm{~g} /$ day (unpublished). The changes in body weight during the training period as summarized in Table 1 indicate that at the end of the training period the trainees were less heavy compared to their sedentary counterparts. Body weight gain in two of the cages that housed 12month-old SE-C (N) and SE-C $(+\mathrm{E})$ as indicated in table 1 were lesser than those of the 8-month-old counterparts over a period of 60 days when they were 10 months of age. This discrepancy in bodyweights between the 8-and 12-monthold sedentary animals will be pursued in all our future colonies with a view to find a possible explanation. Lack of cardiac hypertrophy, as revealed by insignificant changes in $\mathrm{HW} / \mathrm{BW}$ and regional weight $(\mathrm{RW}) / \mathrm{BW}$ ratios was a noticeable feature in the 4-, 8-, and 12-month old animals. In the 22 month-old animals, the trained animals exhibited significant elevation in HW/BW as well as in the regional weights/BW (Table 2).

Table 2

Heart and body weight changes in vitamin-E supplemented and exercisetrained rats

\begin{tabular}{lllll}
\hline $\begin{array}{l}\text { Age } \\
\text { (months) }\end{array}$ & Groups & $\begin{array}{l}\text { HW/BW } \\
(\mathrm{mg} / \mathrm{g})\end{array}$ & $\begin{array}{l}\text { LV/BW } \\
(\mathrm{mg} / \mathrm{g})\end{array}$ & $\begin{array}{l}\text { RV/BW } \\
(\mathrm{mg} / \mathrm{g})\end{array}$ \\
\hline \multirow{4}{*}{4} & & & & \\
& SE-C(N) & $2.50 \pm 0.01$ & $1.86 \pm 0.05$ & $0.38 \pm 0.01$ \\
& SE-C(+E) & $2.49 \pm 0.09$ & $1.87 \pm 0.07$ & $0.39 \pm 0.02$ \\
& SW-T(N) & $2.89 \pm 0.11^{-}$ & $2.19 \pm 0.07$ & $0.37 \pm 0.02$ \\
& SW-T(+E) & $2.87 \pm 0.02^{-}$ & $2.08 \pm 0.01$ & $0.39 \pm 0.02$ \\
& SE-C(N) & $2.56 \pm 0.11$ & $1.24 \pm 0.04$ & $0.81 \pm 0.02$ \\
& SE-C(+E) & $2.37 \pm 0.06^{\circ}$ & $1.12 \pm 0.04$ & $0.75 \pm 0.07$ \\
& SW-T(N) & $2.51 \pm 0.16$ & $1.21 \pm 0.05$ & $0.77 \pm 0.01^{\prime \prime}$ \\
& SW-T(+E) & $2.60 \pm 0.05$ & $1.24 \pm 0.01$ & $0.83 \pm 0.03$ \\
& & & & \\
& SE-C(N) & $3.19 \pm 0.09$ & $1.71 \pm 0.04$ & $0.95 \pm 0.02$ \\
& SE-C(+E) & $3.22 \pm 0.05$ & $1.51 \pm 0.07$ & $0.99 \pm 0.02$ \\
& SW-T(N) & $3.07 \pm 0.03$ & $1.76 \pm 0.15$ & $0.94 \pm 0.03$ \\
& SW-T(+E) & $2.75 \pm 0.06^{\vee}$ & $1.42 \pm 0.07^{\prime \prime}$ & $0.90 \pm 0.07$ \\
& SE-C(N) & $2.31 \pm 0.18$ & $1.28 \pm 0.05$ & $0.74 \pm 0.01$ \\
& SE-C(+E) & $2.22 \pm 0.21$ & $1.39 \pm 0.04$ & $0.61 \pm 0.01$ \\
& SW-T(N) & $2.76 \pm 0.25^{*}$ & $1.65 \pm 0.04^{*}$ & $0.79 \pm 0.03^{-}$ \\
& SW-T(+E) & $2.77 \pm 0.14^{*}$ & $1.67 \pm 0.01^{*}$ & $0.80 \pm 0.01$ \\
\hline
\end{tabular}

Values are mean $\pm \mathrm{SE}$ of three animals/group. Significance of differences: ${ }^{-} P<0.01,{ }^{\circ} P<0.05,{ }^{\vee} P<0.002,{ }^{\prime \prime} P<0.02,{ }^{*} P<0.001$ (Paired $t$-test), with respect to $\mathrm{SE}-\mathrm{C}(\mathrm{N})$ of each age group. SE-C(N), sedentary control; SE-C $(+E)$, sedentary control supplemented with vitamin E; SW-T $(\mathrm{N})$, trained swimmers; SW-T $(+\mathrm{E})$, trained swimmers supplemented with vitamin E. LV, left ventricle; RV, right ventricle; HW, heart weight; BW body weight. 


\section{Effect of vitamin $E$ on swim parameters}

Final $S_{\mathrm{v}}$ values at all ages showed no significant changes between the trained vitamin E supplemented and unsupplemented groups. Age-related changes were evident with an increase in $S_{\mathrm{v}}$ in 4-, 8-, and 12-month-old animals and a decrease in the 22-month animals in comparison to its preceding age group (Fig. 1(a)). $W_{\text {ext }}$, which increased linearly with age showed no significant variations between the trained groups at all ages (Fig. 1(b)).

With age, there was a significant reduction in the endurance capacity of SW-T rats (Fig. 1(c)) and rats belonging to $\mathrm{SW}-\mathrm{T}(\mathrm{N})$ group had lower endurance capacity as compared to those supplemented with vitamin $\mathrm{E}$ as tested through two-way ANOVA and DMRT analyses. However,

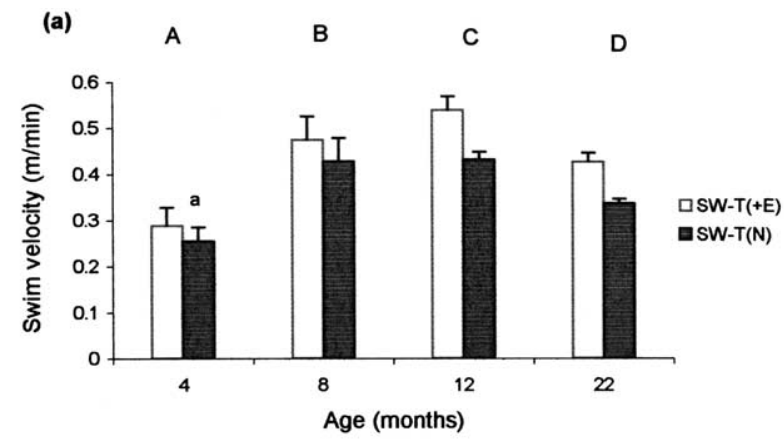

(b)
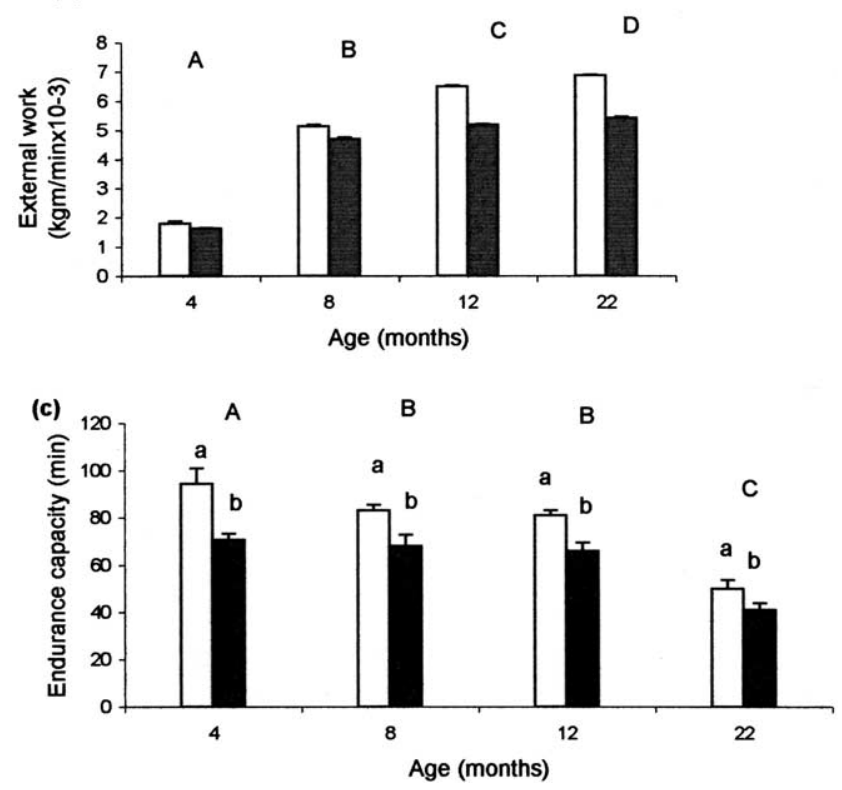

Fig. 1. Results on swim parameters in response to vitamin $E$ and training in rats of different ages. (a) Swim velocity $\left(S_{\mathrm{v}}\right)$. (b) External work done ( $\left.W_{\text {ext }}\right)$. (c) Endurance. Significance between the group means of four ages as analyzed by two-way ANOVA (groups $x$ ages) followed by DMRT is significant at $P<0.05$ and is represented in upper case. Changes were significant between the trained groups of all ages in (c) as represented by the lower case and insignificant in (a) and (b). Those not sharing the same letters are significantly different. Values are mean \pm SE of three animals/group. vitamin E supplemented exercise trainees exhibited higher endurance capacity when compared to their counterparts without vitamin $\mathrm{E}$ supplementation and this was evident in the old too.

Effect of vitamin $E$ and exercise on plasma lipid profile

HDL-C levels exhibited significant increase in both trained groups of all ages over their sedentary counterparts. Concentration of HDL-C decreased from 4-to 8-month, with no change in the 12-and 22-month olds (Fig. 2(a)).

Training was effective in bringing about a decline in LDL-C levels in 4-, 8-, and 12-month-old exercised animals and this was irrespective of vitamin $\mathrm{E}$ supplementation. In the 22-month olds, however, exercise trainees failed to respond to vitamin E supplementation (Fig. 2(b)).

Age-associated increase in cholesterol levels was evident in all the experimental groups. Training was significantly
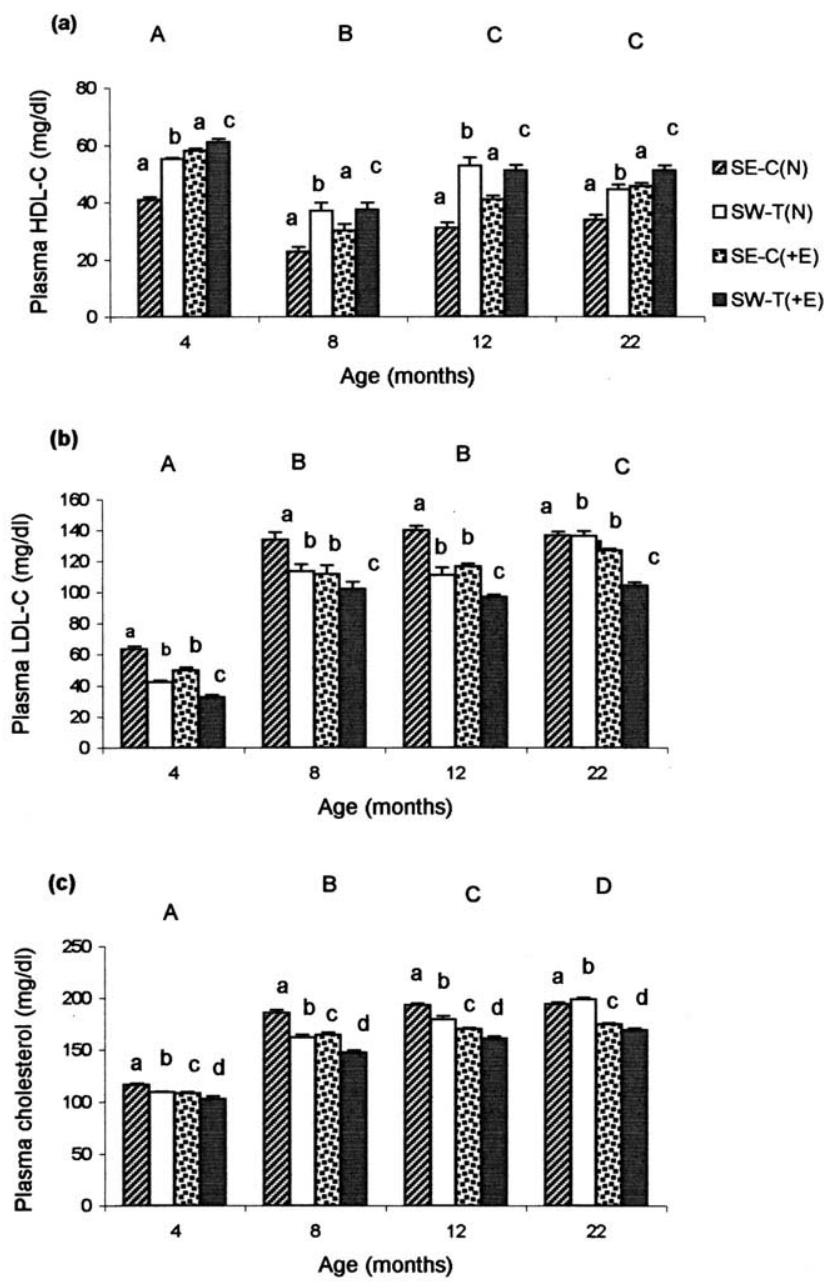

Fig. 2. Plasma lipid profile in animals subjected to exercise training and supplementations of vitamin E. (a) HDL, (b) LDL, (c) cholesterol (C). Significance between the group means of four ages as analyzed by DMRT is at $P<0.05$ and is represented in upper case and between experimental groups in lower case. Those not sharing the same letters are significantly different. Values are mean \pm SE of three animals/group. 
Table 3

Effect of exercise and vitamin $\mathrm{E}$ on plasma total cholesterol/HDL-C ratios in aging rats

\begin{tabular}{lllll}
\hline Groups & \multicolumn{4}{l}{ Age (months) } \\
\cline { 2 - 5 } & 4 & 8 & 12 & 22 \\
\hline SE-C(N) & $2.85 \pm 0.07$ & $5.73 \pm 0.43$ & $6.26 \pm 0.40$ & $5.77 \pm 0.28$ \\
SE-C(+E) & $1.69 \pm 0.03$ & $4.89 \pm 0.54$ & $3.93 \pm 0.18^{*}$ & $3.62 \pm 0.08^{*}$ \\
SW-T(N) & $1.97 \pm 0.01$ & $4.39 \pm 0.37$ & $3.24 \pm 0.20^{*}$ & $4.26 \pm 0.15$ \\
SW-T(+E) & $1.52 \pm 0.05$ & $3.46 \pm 0.36$ & $2.57 \pm 0.17^{*}$ & $3.11 \pm 0.12^{*}$
\end{tabular}

Values are mean $\pm \mathrm{SE}$ of three animals/group. Significance of differences: $P<0.001$ (paired $t$-test) with respect to $\mathrm{SE}-\mathrm{C}(\mathrm{N})$ of each age. SE-C $(\mathrm{N})$, sedentary controls; SE-C $(+\mathrm{E})$, sedentary controls supplemented with vitamin E; SW-T(N), swim trained; SW-T(+E), swim trainees supplemented with vitamin E. HDL-C, high density lipoproteincholesterol.

effective (SW-T) and between the two groups of trainees vitamin $\mathrm{E}$ was more effective in lowering cholesterol levels in all age groups.

Total C/HDL-C ratio was substantially lower in the 12and 22 month-old SW-T (+E) and SW-T (N) rats. However, the reduction was not significant in the 22 month-olds of $\mathrm{SW}-\mathrm{T}(\mathrm{N})$. Although similar reductions were seen in the 4and 8-month olds, the changes were insignificant in relation to their SE-C counterparts (Table 3).

\section{Discussion}

In the present study, swimming was chosen as a suitable mode of exercising rats, since they are good swimmers by nature and are less stressed than when subjected to treadmill running, wherein the animals are constantly under the fear of electrical stimulation (Russell et al., 1980; Sonne and Galbo, 1980). Besides this, the epinephrine levels are very low in swimmers when suitably trained (Benthem et al., 1994; Scheurink et al., 1989; Leblanc et al., 1982). One of the factors that may influence swimming endurance of rodents (Anitha and Asha Devi, 1996) and humans (Yaspelkis et al., 1993) by improving cardiac function either in terms of maximum biochemical or physiological benefits is the medium temperature.

The exercise program employed in this study provided initial brief pre-training swim periods and a gradual lengthening to facilitate swimming and to reduce stress reaction. Lack of hypertrophy as evident from insignificant changes in HW/BW and RW/BW ratios confirms the idea that an increase in cardiac mass is not a sensitive indicator of training effects in rodents (Venditti and Meo, 1996). Vitamin E supplementation has some times been advocated in human and animal models in the hope of improving performance, minimizing exercise-induced muscle damage and maximizing recovery (Tiidus and Houston, 1995). Final $S_{\mathrm{v}}$ values in our study, however, showed no significant changes between the SW-T $(+\mathrm{E})$ and SW-T animals in all age groups, ruling out the possible role of vitamin $\mathrm{E}$ as an ergogenic aid in enhancing performance capacity in the supplemented animals. Exercising capacity, however, decreased with age as evidenced by a lower $S_{\mathrm{v}}$ in the old animals than in the 8 -and 12-month olds. $W_{\text {ext }}$ progressively increased in both the trained groups in all ages. This parameter which is mainly dependent on BW of animals suggests that at no age was the exercise program so stressful as to cause drastic reduction in BW. Endurance capacity was higher in the SW-T $(+\mathrm{E})$ animals of all ages than their trained counterparts without antioxidant supplementation. Our results are in concurrence with those reported on vitamin $\mathrm{E}$ and spin trappers as effective agents in prolonging endurance capacity and fatigue to physical exercise (Novelli et al., 1990), thus supporting the idea that free radicals have a physiological role during exercise. Hence, antioxidant (AO) defenses obtained through exercise and dietary AO manipulation are associated with greater endurance time. Vitamin E, besides keeping free radicals at bay, can stabilize membranes, delay muscle fatigue and thereby enhance endurance (Goldfarb, 1993). The present study on the differential responses in the endurance capacities has indicated that training and AO-induced lengthening of endurance could prove beneficial especially in the older animals.

Positive changes in lipid profile as evidenced through elevated HDL-C, lower LDL-C and total cholesterol in the trained animals strongly emphasize the role of exercise especially in the 4-, 8-, and 12-month-old animals. The changes in concentration of LDL-C in the plasma may imply either an altered synthesis of LDL-C or removal rate of LDL-C from the plasma by the tissues. Exercise bouts of short duration are reported to have negligible impact on total cholesterol and LDL molecules in human subjects (Durstine and Haskell, 1994). Although exercise HDL-C levels are inversely related to coronary heart disease (CHD) risks, the ratio of total cholesterol to HDL-C is considered a better indicator (Willett, 1994). Lower ratios were observed in the trained animals of both vitamin E supplemented and normal diet fed groups. However, vitamin $\mathrm{E}$ has no remarkable effect on lowering the ratio in the 4-and 8-month-olds. Middle-aged SW-T animals exhibited 50\% reductions in the total cholesterol: HDL-C ratio as compared to the SE-C, underlining the significance of exercise. However, in the old, the effects of training alone were obscure, while vitamin E supplementation proved very promising in lowering the ratio. LDL is protected against oxidation by its endogenous AO, primarily $\alpha$-tocopherol (Jialal et al., 1995). One LDL particle contains an average of six molecules of $\alpha$ tocopherol (Esterbauer et al., 1991). Also normal LDL, i.e. without vitamin $\mathrm{E}$ supplementation does not offer oxidative resistance (Jessup et al., 1990). Thus enrichment of LDL particles with vitamin E prevents oxidation of LDL, thereby, reducing the risks of several related pathological conditions.

In conclusion, the data from the present study indicates that in rats, vitamin $\mathrm{E}$ results in better endurance capacity in 
swim trainees. Further, the data demonstrate that prior supplementation by vitamin $\mathrm{E}$ is beneficial in altering the plasma lipid profile and old animals in particular, may benefit from it.

\section{Acknowledgements}

The present study was supported in part by a Grant-inAid from the University Grants Commission, New Delhi, India, (SR-II) to S. Asha Devi. We wish to thank Mr. Ravikiran for technical assistance and Mr. A.K. Goyal for statistical analyses.

\section{References}

Anitha, V., Asha Devi, S., 1996. Age-related responses of right ventricle in swim-trained rats: changes in lactate and pyruvate contents and lactate dehydrogenase activity. Mech. Ageing Dev. 90, 91-102.

Benthem, L., Bolhuis, J.W., Leest, J.V., Stefens, A.B., Zock, J.P., Zulstra, W.G., 1994. Methods for measurement of energy expenditure and substrate concentrations in swimming rats. Physiol. Behav. 56, $151-159$.

Bowry, V.W., Ingold, K.U., Stockler, R., 1992. Vitamin E in human lowdensity lipoprotein. Biochem. J. 288, 341-344.

Brooks, G.A., White, T.P., 1978. Determination of metabolic and heart rate responses of treadmill exercise. J. Appl. Physiol. 45, 1009-1015.

Chan, A.C., 1998. Vitamin E in atherosclerosis. J. Nutr. 128, 1593-1596.

Dawson, C.A., Nadel, E., Hovarth, S.M., 1968. Cardiac output in the cold stressed swimming rats. Am. J. Physiol. 214, 320-325.

Durstine, J.L., Haskell, W.L., 1994. Effects of exercise training on plasma lipids and lipoproteins. In: Holloszy, J.O., (Ed.), Exercise and Sports Science Reviews, vol. 22. Williams and Wilkens, Baltimore, pp. $477-521$

Esterbauer, H., Dieber-Rothen, M., Striegl, G., Waeg, G., 1991. Role of vitamin $\mathrm{E}$ in preventing oxidation of LDL. Am. J. Clin. Nutr. 553, 314S-321S.

Goldfarb, A.H., 1993. Antioxidants: role of supplementation to prevent exercise-induced oxidative stress. Med. Sci. Sports Exerc. 25, 232-236.
Jessup, V., Rankin, S.M., De Whalley, C.V., Hoult, J.R.S., Scott, J., Leake, D.S., 1990. $\alpha$-Tocopherol consumption during low-density lipoprotein oxidation. Biochem. J. 265, 399-405.

Jialal, I., Fuller, C.J., Huct, B.A., 1995. The effect of alpha-tocopherol supplementation on low-density lipoprotein oxidation. A dose response study. Arterioscler. Thromb. Vasc. Biol. 15, 190-194.

Leblanc, J., Dussault, J., Lupien, D., Richard, D., 1982. Effect of diet and exercise on norepinephrine-induced thermogenesis in male and female rats. J. Appl. Physiol. 52, 556-561.

Novelli, G.P., Bracciotti, G., Falsini, S., 1990. Spin-trappers and vitamin E prolong endurance to muscle fatigue in mice. Free Rad. Biol. Med. 8, 9-13.

Polidori, M.C., Mccocci, P., Cherubin, A., Senin, U., 2000. Physical activity and oxidative stress during aging. Int. J. Sports Med. 21, 154-157.

Prathima, S., Asha Devi, S., 1999. Adaptations in lactose dehydrogenase and its isoenzymes in aging myocardium: interaction of exercise and temperature. Mech. Ageing Dev. 108, 61-75.

Russell, J.C., Campagna, P.D., Wenger, H.A., 1980. Small-animal ergometer. J. Appl. Physiol. 48, 394-398.

Scheurink, A.J.W., Stefens, A.B., Dreteler, G.H., Bentham, L., Bruntnik, R., 1989. Experience affects exercise-induced changes in catecholamines, glucose, and FFA. Amm. J. Physiol. 256, R169-R173.

Sharon, C., Asha Devi, S., 2001. Myosin ATPase in young and old mammalian myocardium: responses to exercise in cold and thermo neutral environment. Ind. J. Exp. Biol. 39, 29-34.

Snedecor, G.W, Cochran, W.G, 1994. Statistical Methods, Iowa State University Press, Amesterdam.

Sonne, B., Galbo, H., 1980. Simultaneous determination of metabolic and harmaonal responses, heart rate, temperature and oxygen uptake in running rats. Acta Physiol. Scand. 109, 201-209.

Tiidus, P.M, Houston, E., 1995. Vitamin E status and response to exercise training. Sports Med. 20, 12-23.

VanDer Beek, E.I., 1991. Vitamin supplementation and physical exercise performance. J. Sport Sci. 9, 77-89.

Venditti, P., Meo, S.D., 1996. Antioxidants, tissue damage, and endurance in trained and untrained young male rats. Arch. Biochem. Biophys. 331, $63-66$.

Willett, W.C., 1994. Diet and health. What should we eat? Science 264, 532-537.

Yaspelkis, B.B., Scroop, G.C., Wilmore, K.M., Ivy, J.L., 1993. Carbohydrate metabolism during exercise in hot and thermo neutral environments. Int. J. Sports Med. 14, 13-20. 